\title{
Does full imply faithful?
}

\author{
Alberto Canonaco, Dmitri Orlov, and Paolo Stellari*
}

\begin{abstract}
We study full exact functors between triangulated categories. With some hypotheses on the source category we prove that it admits an orthogonal decomposition into two pieces such that the functor restricted to one of them is zero while the restriction to the other is faithful. In particular, if the source category is either the category of perfect complexes or the bounded derived category of coherent sheaves on a noetherian scheme supported on a closed connected subscheme, then any non-trivial exact full functor is faithful as well. Finally we show that removing the noetherian hypothesis this result is not true.
\end{abstract}

Mathematics Subject Classification (2010). 14F05, 18E10, 18E30.

Keywords. Derived categories, triangulated categories, exact functors.

\section{Introduction}

For an exact functor $\mathrm{F}: \mathbf{T}_{1} \rightarrow \mathbf{T}_{2}$ between triangulated categories, there is a list of properties that, from a purely categorical point of view, are completely unrelated or not automatically satisfied. Among them we can mention: the existence of adjoints, fullness, faithfulness and essential surjectivity. Nevertheless, as soon as $\mathbf{T}_{i}$ has a geometric nature, these properties and their relations can be studied in a more efficient and complete way.

For example, if $\mathbf{T}_{i}$ is the bounded derived category $\mathrm{D}^{\mathrm{b}}\left(X_{i}\right)$ of coherent sheaves on a complex smooth projective variety $X_{i}$, then any exact functor $\mathrm{F}: \mathrm{D}^{\mathrm{b}}\left(X_{1}\right) \rightarrow \mathrm{D}^{\mathrm{b}}\left(X_{2}\right)$ has always a left and a right adjoint, by a result of Bondal and Van den Bergh [3]. This combined with [9] says that if $\mathrm{F}$ is fully faithful, then it is of Fourier-Mukai type, i.e., there is $\mathcal{E} \in \mathrm{D}^{\mathrm{b}}\left(X_{1} \times X_{2}\right)$ and an isomorphism of functors $\mathrm{F} \cong \Phi_{\mathcal{E}}$, where $\Phi_{\varepsilon}: \mathrm{D}^{\mathrm{b}}\left(X_{1}\right) \rightarrow \mathrm{D}^{\mathrm{b}}\left(X_{2}\right)$ is the exact functor defined by

$$
\Phi_{\mathcal{E}}:=\mathbf{R}\left(p_{2}\right)_{*}\left(\mathcal{E} \otimes^{\mathbf{L}} p_{1}^{*}(-)\right),
$$

and $p_{i}: X_{1} \times X_{2} \rightarrow X_{i}$ is the natural projection.

\footnotetext{
*The second author was partially supported by RFBR grants 11-01-00336, 11-01-00568, NSh grant 5139.2012.1, by AG Laboratory HSE, RF gov. grant, ag. 11.G34.31.0023. The third author was partially supported by the MIUR of the Italian Government in the framework of the National Research Project "Geometria algebrica e aritmetica, teorie coomologiche e teoria dei motivi" (PRIN 2008).
} 
Now [2] and [4] provide a very useful criterion to establish when a Fourier-Mukai functor $\Phi_{\mathcal{E}}: \mathrm{D}^{\mathrm{b}}\left(X_{1}\right) \rightarrow \mathrm{D}^{\mathrm{b}}\left(X_{2}\right)$ is fully faithful. Namely $\Phi_{\mathcal{E}}$ is such if and only if

$$
\operatorname{Hom}_{\mathrm{D}^{\mathrm{b}}\left(X_{2}\right)}\left(\Phi_{\mathcal{E}}\left(\mathcal{O}_{x_{1}}\right), \Phi_{\mathcal{E}}\left(\mathcal{O}_{x_{2}}\right)[i]\right) \cong \begin{cases}\mathbb{C} & \text { if } x_{1}=x_{2} \text { and } i=0, \\ 0 & \text { if } x_{1} \neq x_{2} \text { or } i \notin\left[0, \operatorname{dim} X_{1}\right],\end{cases}
$$

for all closed points $x_{1}, x_{2} \in X_{1}$.

Of course, it is quite easy to construct examples of faithful functors which are not full (e.g. the tensorization by a vector bundle of rank greater than 1). On the other hand, using all the remarks above and a collection of standard results, it is not difficult to see that a non-trivial full exact functor $\mathrm{F}: \mathrm{D}^{\mathrm{b}}\left(X_{1}\right) \rightarrow \mathrm{D}^{\mathrm{b}}\left(X_{2}\right)$ is also faithful. Here we give a sketch of the proof, since a more general statement will be proved in the paper. First, by the main result of [5] (that improves [9]), F is a Fourier-Mukai functor. Thus, because of the above criterion and the fact that $\mathrm{F}$ is full, to show that the functor is also faithful it is enough to prove that there are no closed points $x \in X_{1}$ such that $\operatorname{Hom}\left(\mathrm{F}\left(\mathcal{O}_{x}\right), \mathrm{F}\left(\mathcal{O}_{x}\right)\right)=0$ or, in other words, such that $\mathrm{F}\left(\mathcal{O}_{x}\right) \cong 0$. To see this, take the left adjoint $\mathrm{G}: \mathrm{D}^{\mathrm{b}}\left(X_{2}\right) \rightarrow \mathrm{D}^{\mathrm{b}}\left(X_{1}\right)$ of $\mathrm{F}$ and consider the composition $\mathrm{G} \circ \mathrm{F}$ which is again a Fourier-Mukai functor, hence isomorphic to $\Phi_{\mathcal{E}}$ for some $\mathcal{E} \in \mathrm{D}^{\mathrm{b}}\left(X_{1} \times X_{1}\right)$. Assume that there are $x_{1}, x_{2} \in X_{1}$ such that $\mathrm{F}\left(\mathcal{O}_{x_{1}}\right) \not 0$ while $\mathrm{F}\left(\mathcal{O}_{x_{2}}\right) \cong 0$. By [2] (see, in particular, Proposition 1.5 there) the Chern character $\operatorname{ch}\left(\Phi_{\mathcal{E}}\left(\mathcal{O}_{x_{1}}\right)\right)$ is not zero. On the other hand, it is proved in [9] that the functor $\Phi_{\mathcal{E}}$ induces a morphism $\Phi_{\varepsilon}^{H}: H^{*}\left(X_{1}, \mathbb{Q}\right) \rightarrow H^{*}\left(X_{1}, \mathbb{Q}\right)$ such that

$$
\begin{aligned}
0 \neq \operatorname{ch}\left(\Phi_{\mathcal{E}}\left(\mathcal{O}_{x_{1}}\right)\right) \cdot \sqrt{\operatorname{td}\left(X_{2}\right)} & =\Phi_{\mathcal{E}}^{H}\left(\operatorname{ch}\left(\mathcal{O}_{x_{1}}\right) \cdot \sqrt{\operatorname{td}\left(X_{1}\right)}\right) \\
& =\Phi_{\mathcal{E}}^{H}\left(\operatorname{ch}\left(\mathcal{O}_{x_{2}}\right) \cdot \sqrt{\operatorname{td}\left(X_{1}\right)}\right)=0,
\end{aligned}
$$

where td denotes the Todd class. This contradiction proves that if $\mathrm{F}$ were not faithful, then $\mathrm{F}\left(\mathcal{O}_{x}\right) \cong 0$ for every closed point $x \in X$. But this would easily imply that $\mathrm{F} \cong 0$, against the assumption.

This paper is an attempt to understand to which extent the previous easy example can be pushed. In particular, we want to study when the following question may have a positive answer:

When is a full exact functor between 'geometric triangulated categories' faithful?

It is rather obvious that one can produce examples of full non-trivial exact functors which are not faithful if one does not require the source triangulated category to be indecomposable. However, something interesting can be said even without this hypothesis. In fact, after proving a very general statement in Section 2, our first important (and still rather general) result, whose proof is in Section 3, is the following.

Theorem 1.1. Let $\mathbf{T}_{1}$ be a triangulated category with arbitrary direct sums which is compactly generated and let $\mathbf{T}_{1}^{\mathrm{c}}$ be the subcategory of compact objects. Let $S \subset \mathbf{T}_{1}^{\mathrm{c}}$ 
be a subset of compact objects and let $\mathbf{S} \subseteq \mathbf{T}_{1}^{\mathrm{c}}$ be the thick subcategory generated by S. Let

$$
\mathrm{F}: \mathrm{S} \rightarrow \mathbf{T}_{2}
$$

be a full exact functor to a triangulated category $\mathbf{T}_{2}$. Assume that for any object $A \in S$ the ring of endomorphisms $\operatorname{End}_{\mathbf{T}_{1}}(A)$ is idempotent noetherian. Then there is an orthogonal decomposition

$$
\mathbf{S}=(\operatorname{ker} F)^{\perp} \oplus \operatorname{ker} F
$$

and $\left.\mathrm{F}\right|_{(\mathrm{ker} F)^{\perp}}$ is faithful.

See Definition 3.1 for the notion of idempotent noetherian ring. As it will turn out, the ring of endomorphisms of an object in the bounded derived category of coherent sheaves on a noetherian scheme has this property (see Proposition 4.3).

Notice that if in Theorem 1.1 we assume $\mathbf{S}$ to be indecomposable and $\mathrm{F}$ to be non-trivial, then we can conclude that $F$ is actually faithful. So in the geometric case we consider a noetherian scheme $X$ containing a closed connected subscheme $Z$ and we assume that $\mathbf{S}$ is either the bounded derived category $\mathrm{D}_{Z}^{\mathrm{b}}(X)$ of coherent sheaves on $X$ supported on $Z$ or the subcategory $\operatorname{Perf}_{Z}(X) \subseteq \mathrm{D}_{Z}^{\mathrm{b}}(X)$ consisting of perfect complexes. Recall that a complex in $\mathrm{D}_{Z}^{\mathrm{b}}(X)$ is perfect if it is locally quasi-isomorphic to a complex of locally free sheaves of finite type on $X$. Due to Corollary 4.6, these categories are indecomposable, and we get the following result which we prove in Section 4.

Theorem 1.2. Let $X$ be a noetherian scheme containing a closed subscheme $Z$ and let $\mathbf{S}$ be either $\operatorname{Perf}_{Z}(X)$ or $\mathrm{D}_{Z}^{\mathrm{b}}(X)$. Let $\mathbf{T}$ be a triangulated category and let

$$
\mathrm{F}: \mathbf{S} \rightarrow \mathbf{T}
$$

be a full exact functor which is not isomorphic to the zero functor. If $Z$ is connected, then $\mathrm{F}$ is also faithful.

In Section 5 we show that if we do not assume $X$ to be noetherian, then the above result does not necessarily hold true. Indeed, we give an example of a non-noetherian (affine) scheme $X$ over a field $\mathbb{k}$ such that $\operatorname{Perf}(X)$ is indecomposable and of a full non-trivial exact functor $\mathrm{F}: \operatorname{Perf}(X) \rightarrow \mathbf{D}(\mathbb{k})$ to the (unbounded) derived category of $k$-vector spaces which is not faithful.

\section{A general result}

If $\mathrm{F}: \mathbf{A} \rightarrow \mathbf{B}$ is an additive functor between additive categories, we will denote by ker $\mathrm{F}$ the (strictly) full subcategory of $\mathbf{A}$ having as objects the $A$ such that $\mathrm{F}(A) \cong 0$, and by im $\mathbf{F}$ the (strictly) full subcategory of $\mathbf{B}$ having as objects the $B$ such that 
$B \cong \mathrm{F}(A)$ for some $A \in \mathbf{A}$. Notice that ker $\mathrm{F}$ is a (thick) triangulated subcategory of $\mathbf{A}$ if $\mathbf{A}$ and $\mathbf{B}$ are triangulated and $\mathbf{F}$ is exact.

For the convenience of the reader we recall the proof of the following lemma which is known to experts and, for example, is contained in the proof of [10], Thm. 3.9.

Lemma 2.1. Let $\mathbf{T}_{1}$ and $\mathbf{T}_{2}$ be triangulated categories and let $\mathbf{F}: \mathbf{T}_{1} \rightarrow \mathbf{T}_{2}$ be a full exact functor such that $\mathrm{ker} \mathrm{F} \cong 0$. Then $\mathrm{F}$ is faithful.

Proof. Assume that there are $A, B \in \mathbf{T}_{1}$ and $f: A \rightarrow B$ a morphism such that $\mathbf{F}(f)=0$. Complete the morphism to a distinguished triangle

$$
A \stackrel{f}{\longrightarrow} B \stackrel{g}{\longrightarrow} \mathrm{C}(f)
$$

so that, applying the functor $F$, we get the distinguished triangle

$$
\mathrm{F}(A) \stackrel{\mathrm{F}(f)=0}{\longrightarrow} \mathrm{F}(B) \stackrel{\mathrm{F}(g)}{\longrightarrow} \mathrm{F}(\mathrm{C}(f)) .
$$

Then id: $\mathrm{F}(B) \rightarrow \mathrm{F}(B)$ factors through $\mathrm{F}(g): \mathrm{F}(B) \rightarrow \mathrm{F}(\mathrm{C}(f))$.

As $\mathrm{F}$ is full, there exists a morphism $h: B \rightarrow B$ factoring through $g$ and such that $\mathrm{F}(h)=$ id. Then $\mathrm{F}(\mathrm{C}(h)) \cong \mathrm{C}(\mathrm{F}(h)) \cong 0$. Since ker $\mathrm{F} \cong 0$, we get $\mathrm{C}(h) \cong 0$ and $h$ is an isomorphism. This implies that $g$ is a (split) monomorphism. In particular $f=0$, and so $\mathrm{F}$ is faithful.

Definition 2.2. An orthogonal decomposition $\mathbf{T}=\mathbf{T}_{1} \oplus \mathbf{T}_{2}$ of a triangulated category $\mathbf{T}$ is given by two full triangulated subcategories $\mathbf{T}_{1}$ and $\mathbf{T}_{2}$ satisfying the following conditions:

(1) $\mathbf{T}_{1}$ and $\mathbf{T}_{2}$ are completely orthogonal, meaning that

$$
\operatorname{Hom}\left(A_{1}, A_{2}\right)=\operatorname{Hom}\left(A_{2}, A_{1}\right)=0
$$

for every objects $A_{i}$ of $\mathbf{T}_{i}$;

(2) for every object $A$ of $\mathbf{T}$ there exist objects $A_{i}$ of $\mathbf{T}_{i}$ such that $A \cong A_{1} \oplus A_{2}$.

A triangulated category is indecomposable if it admits only trivial orthogonal decompositions.

We begin with the following general result.

Proposition 2.3. Let $\mathbf{T}_{1}$ and $\mathbf{T}_{2}$ be triangulated categories and let $\mathbf{F}: \mathbf{T}_{1} \rightarrow \mathbf{T}_{2}$ be a full exact functor. Assume moreover that the projection functor $\pi: \mathbf{T}_{1} \rightarrow$ $\mathbf{T}_{1} /$ ker $\mathbf{F}$ has an adjoint $\mu: \mathbf{T}_{1} / \operatorname{ker} \mathbf{F} \rightarrow \mathbf{T}_{1}$. Then the category $\mathbf{T}_{1}$ has an orthogonal decomposition of the form

$$
\mathbf{T}_{1}=\operatorname{im} \mu \oplus \operatorname{ker} \mathrm{F}
$$

and $\left.\mathrm{F}\right|_{\operatorname{im} \mu}$ is faithful. In particular, if $\mathbf{T}_{1}$ is indecomposable and $\mathrm{F}$ is not isomorphic to 0 , then $\mathrm{F}$ is faithful. 
Proof. Passing, if necessary, to the opposed functor of $\mathrm{F}$ (defined as $\mathrm{F}$, but between the opposed categories), we can assume that $\mu$ is a right adjoint of $\pi$.

Now, given $A \in \mathbf{T}_{1}$ and using the adjunction between $\mu$ and $\pi$, we get the distinguished triangle

$$
A \stackrel{m_{A}}{\longrightarrow} \mu \circ \pi(A) \stackrel{n_{A}}{\longrightarrow} N_{A} .
$$

The functor $\mathrm{F}$ induces in a natural way a functor $\mathrm{F}^{\prime}: \mathbf{T}_{1} / \operatorname{ker} \mathrm{F} \rightarrow \mathbf{T}_{2}$ which is fully faithful due to Lemma 2.1. Hence, for all $A, B \in \mathbf{T}_{1}$,

$$
\begin{aligned}
\operatorname{Hom}(B, \mu \circ \pi(A)) & \cong \operatorname{Hom}(\pi(B), \pi(A)) \\
& \cong \operatorname{Hom}\left(\mathrm{F}^{\prime} \circ \pi(B), \mathrm{F}^{\prime} \circ \pi(A)\right) \\
& =\operatorname{Hom}(\mathrm{F}(B), \mathrm{F}(A)) .
\end{aligned}
$$

As $\mathrm{F}=\mathrm{F}^{\prime} \circ \pi$ is full, this implies that the morphism

$$
\operatorname{Hom}(B, A) \rightarrow \operatorname{Hom}(B, \mu \circ \pi(A))
$$

given by the composition with $m_{A}$ is surjective for all $A, B \in \mathbf{T}_{1}$. In particular, the map

$$
\varphi_{A, B}: \operatorname{Hom}(B, \mu \circ \pi(A)) \rightarrow \operatorname{Hom}\left(B, N_{A}\right),
$$

obtained composing with $n_{A}$, is zero. Taking $B=\mu \circ \pi(A)$ in the above argument, we get $\varphi_{A, B}$ (id) $=n_{A}=0$. This means that, for any $A \in \mathbf{T}_{1}$, there is a decomposition

$$
A \cong \mu \circ \pi(A) \oplus N_{A}[-1] .
$$

By [8], Lemma 9.1.7, the functor $\mu$ as adjoint to a projection functor is fully faithful, i.e. $\pi \circ \mu \cong$ id. Therefore, the functor $\pi$ induces an equivalence between $\operatorname{im} \mu$ and the quotient $\mathbf{T}_{1} /$ ker $\mathbf{F}$. Since $\mathbf{F}^{\prime}$ is faithful, the functor $\left.\mathbf{F}\right|_{\operatorname{im} \mu}$ is faithful too.

Moreover, since $\mu$ is fully faithful the map $\pi\left(m_{A}\right)$ is an isomorphism. This implies that $\pi\left(N_{A}\right) \cong \mathrm{C}\left(\pi\left(m_{A}\right)\right) \cong 0$. In order to get the orthogonal decomposition, it remains to show that $\operatorname{ker} \mathrm{F}$ and $\operatorname{im} \mu=\operatorname{im}(\mu \circ \pi)$ are orthogonal. By adjunction, it is obvious that $\operatorname{Hom}(A, B)=0$ if $A \in \operatorname{ker} \mathrm{F}$ and $B \in \operatorname{im} \mu$. For the other direction, assume that there is a morphism $f: \mu \circ \pi(A) \rightarrow B$, for some $A \in \mathbf{T}_{1}$ and $B \in \operatorname{ker} \mathrm{F}$. Consider the distinguished triangle

$$
\mu \circ \pi(A) \stackrel{f}{\longrightarrow} B \rightarrow \mathrm{C}(f)
$$

and apply the functor $\pi$ getting

$$
\pi \circ \mu \circ \pi(A) \stackrel{\pi(f)}{\longrightarrow} \pi(B) \rightarrow \pi(\mathrm{C}(f)) .
$$

Thus $\pi(\mathrm{C}(f)[-1]) \cong \pi \circ \mu \circ \pi(A) \cong \pi(A)$ and $\mu \circ \pi(\mathrm{C}(f)[-1]) \cong \mu \circ \pi(A)$. Moreover the map $\mathrm{C}(f)[-1] \rightarrow \mu \circ \pi(A)$ can be identified with the canonical map $\mathrm{C}(f)[-1] \rightarrow \mu \circ \pi(\mathrm{C}(f)[-1])$.

Because $\mathrm{C}(f) \in \mathbf{T}_{1}$, the calculations above imply that the map $\mathrm{C}(f)[-1] \rightarrow$ $\mu \circ \pi(\mathrm{C}(f)[-1]) \cong \mu \circ \pi(A)$ is an epimorphism, and so $f=0$. This is what we need to prove. 
Remark 2.4. It is well known that every exact functor from $\mathbf{T}_{1}$ has a right (respectively left) adjoint if $\mathbf{T}_{1}$ is right (respectively left) saturated (see [3]).

Remark 2.5. Assume that $\mathbf{T}_{1}$ and $\mathbf{T}_{2}$ are triangulated categories and let $F: \mathbf{T}_{1} \rightarrow \mathbf{T}_{2}$ be a full exact functor admitting a pseudo-adjoint $G: \mathbf{T}_{2} \rightarrow \widetilde{\mathbf{T}}_{1}$ such that $\mathrm{im}(\mathrm{G} \circ \mathrm{F}) \subseteq$ $\mathbf{T}_{1}$. Then $\pi$ has an adjoint which is simply $G \circ F^{\prime}$ (where $F^{\prime}: \mathbf{T}_{1} / \operatorname{ker} F \rightarrow \mathbf{T}_{2}$ is as in the above proof). Hence Proposition 2.3 applies.

With a left (respectively right) pseudo-adjoint of a functor $\mathrm{F}: \mathbf{C} \rightarrow \mathbf{C}^{\prime}$ we mean a functor $\mathrm{G}: \mathbf{C}^{\prime} \rightarrow \widetilde{\mathbf{C}}$, where $\widetilde{\mathbf{C}}$ is some category containing $\mathbf{C}$ as a full subcategory, together with a natural isomorphism $\operatorname{Hom}_{\mathrm{C}^{\prime}}\left(A^{\prime}, \mathrm{F}(A)\right) \cong \operatorname{Hom}_{\widetilde{\mathbf{C}}}\left(\mathrm{G}\left(A^{\prime}\right), A\right)$ (respectively $\operatorname{Hom}_{\mathbf{C}^{\prime}}\left(\mathrm{F}(A), A^{\prime}\right) \cong \operatorname{Hom}_{\widetilde{\mathbf{C}}}\left(A, \mathrm{G}\left(A^{\prime}\right)\right)$ ) for every object $A$ of $\mathbf{C}$ and $A^{\prime}$ of $\mathbf{C}^{\prime}$.

\section{The categorical case}

In this section we prove Theorem 1.1 and show how to apply it to subcategories of noetherian objects. For this purpose we introduce the notion of idempotent noetherian ring.

3.1. General setting. We will be interested in the following special class of rings appearing naturally in geometric situations.

Definition 3.1. A ring $R$ is (right) idempotent noetherian if for every sequence $\left\{a_{i}\right\}_{i \in \mathbb{N}}$ of elements in $R$ satisfying

$$
a_{j} a_{i}=a_{i} \quad \text { for all } i<j
$$

there exists a positive integer $n$ such that $a_{i} R=a_{n} R$ for all $i \geq n$.

Analogously, one can define left idempotent noetherian rings. As this notion will not be needed in the rest of the paper, right idempotent noetherian rings will simply be called idempotent noetherian.

Remark 3.2. If $\left\{a_{i}\right\}_{i \in \mathbb{N}}$ is a sequence in a ring $R$ satisfying (3.1) and such that $a_{i} R=a_{n} R$ for $i \geq n$, then $a_{i}$ is idempotent for $i>n$. Indeed, there exists $r \in R$ such that $a_{i}=a_{i-1} r$, hence

$$
a_{i} a_{i}=a_{i} a_{i-1} r=a_{i-1} r=a_{i} .
$$

We begin with the following easy result.

Lemma 3.3. If $\mathbf{A}$ is an additive category and $A \in \mathbf{A}$ is such that $\operatorname{End}_{\mathbf{A}}(A)$ is idempotent noetherian, then $A$ is isomorphic to a finite direct sum of indecomposable objects. 
Proof. Assume on the contrary that $A$ is not isomorphic to a finite direct sum of indecomposable objects. Then there exists a sequence $\left\{A_{i}\right\}_{i \in \mathbb{N}}$ of non-trivial objects of A such that, for all $n \in \mathbb{N}$, the object $B_{n}:=\bigoplus_{j=0}^{n} A_{j}$ is a direct summand of $A$. Thus, for all $j \in \mathbb{N}$, let $a_{j} \in \operatorname{End}(A)$ be the projection onto $B_{j}$. Clearly the sequence $\left\{a_{i}\right\}_{i \in \mathbb{N}}$ satisfies (3.1), but the ascending chain of right ideals generated by the $a_{i}$ 's does not stabilize.

As a matter of notation, recall that if $\mathbf{T}$ is a triangulated category with arbitrary direct sums and $S$ is a set of objects of $\mathbf{T}$, the localizing subcategory generated by $S$ is the smallest strictly full triangulated subcategory of $\mathbf{T}$ containing $S$ and closed under arbitrary direct sums.

An object $A$ in a triangulated category $\mathbf{T}$ admitting arbitrary direct sums is called compact if, for each family of objects $\left\{X_{i}\right\}_{i \in I} \subset \mathbf{T}$, the canonical map

$$
\bigoplus_{i} \operatorname{Hom}\left(A, X_{i}\right) \rightarrow \operatorname{Hom}\left(A, \oplus_{i} X_{i}\right)
$$

is an isomorphism. The triangulated category $\mathbf{T}$ is compactly generated if there is a set $S$ of compact objects such that $E \in \mathbf{T}$ vanishes if $\operatorname{Hom}(A, E[i])=0$ for all $A \in S$ and all $i \in \mathbb{Z}$. For more details, the reader may consult [12], Sect. 3.1.

Proof of Theorem 1.1. Denote by $\langle S\rangle \subseteq \mathbf{T}_{1}$ the localizing subcategory generated by the set $S$. This category admits arbitrary direct sums and is compactly generated too. Moreover, it is known that the subcategory of its compact objects $\langle S\rangle^{\mathrm{c}}$ coincides with $\mathbf{S}$ (see [7], Lemma 2.2). Hence, replacing $\mathbf{T}_{1}$ with $\langle S\rangle$ we can assume that $\mathbf{T}_{1}$ is compactly generated by the set $S$ and $\mathbf{S}=\mathbf{T}_{1}^{\mathrm{c}}$.

Denote by $\langle\operatorname{ker} F\rangle \subseteq \mathbf{T}_{1}$ the localizing subcategory that is generated by the set of compact objects from ker F. By [7], Thm. 2.1, the canonical functor $\mathbf{T}_{1}^{\mathrm{c}} / \operatorname{ker} \mathbf{F} \rightarrow$ $\mathbf{T}_{1} /\langle\operatorname{ker} \mathrm{F}\rangle$ is fully faithful and its essential image is the subcategory $\left(\mathbf{T}_{1} /\langle\operatorname{ker} F\rangle\right)^{\mathrm{c}}$. As $\mathbf{T}_{1}$ is compactly generated the projection $\pi: \mathbf{T}_{1} \rightarrow \mathbf{T}_{1} /\langle\operatorname{ker} \mathrm{F}\rangle$ has a fully faithful right adjoint $\mu: \mathbf{T}_{1} /\langle\operatorname{ker} \mathbf{F}\rangle \rightarrow \mathbf{T}_{1}$ (see Theorem 8.4.4 and Lemma 9.1.7 in [8]).

By Proposition 2.3, the result is proved if $\mu \circ \pi(A)$ is compact for any compact $A \in \mathbf{T}_{1}^{\mathrm{c}}$. Since $\mathbf{T}_{1}^{\mathrm{c}}$ is the smallest thick subcategory containing $S$, it is enough to prove that $\mu \circ \pi(A) \in \mathbf{T}_{1}^{\mathrm{c}}$ for any $A \in S$. In view of Lemma 3.3, we can assume that $A$ is indecomposable.

Consider the adjunction morphism $m_{A}: A \rightarrow \mu \circ \pi(A)$ and complete it to a distinguished triangle

$$
N_{A}[-1] \stackrel{l_{A}}{\longrightarrow} A \stackrel{m_{A}}{\longrightarrow} \mu \circ \pi(A) \stackrel{n_{A}}{\longrightarrow} N_{A} .
$$

Of course, the result is proved if we show that $n_{A}$ is the zero map, whence we can assume that $N_{A} \not 0$.

The functor $F$ is full and so, by the same argument as in the proof of Proposition 2.3, the map $\operatorname{Hom}_{\mathbf{T}_{1}}(B, A) \stackrel{m_{A^{\circ}(-)}}{\longrightarrow} \operatorname{Hom}_{\mathbf{T}_{1}}(B, \mu \circ \pi(A))$ is surjective for any compact 
object $B \in \mathbf{T}_{1}^{\mathrm{c}}$. This implies that the map

$$
\operatorname{Hom}_{\mathbf{T}_{1}}(B, \mu \circ \pi(A)) \stackrel{n_{A^{\circ}(-)}}{\longrightarrow} \operatorname{Hom}_{\mathbf{T}_{1}}\left(B, N_{A}\right)
$$

is zero.

Since $\mathbf{T}_{1}$ is compactly generated, there exists $Z \in \mathbf{T}_{1}^{\mathrm{c}}$ and a non-trivial morphism $\phi_{0}: Z \rightarrow N_{A}[-1]$. Denote by $C_{Z}$ the cone in $\mathbf{T}_{1}^{\mathrm{c}}$ of the morphism $l_{A} \circ \phi_{0}: Z \rightarrow A$ and consider the commutative diagram whose rows are distinguished triangles:

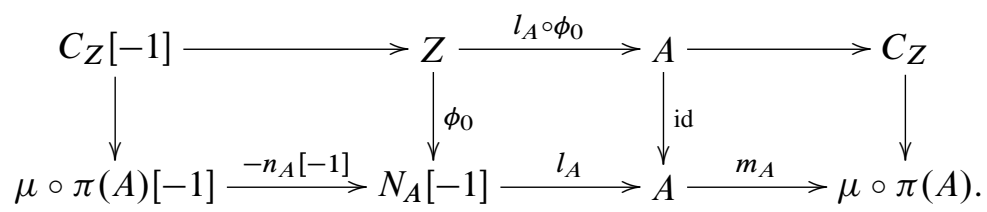

Being $C_{Z}$ a compact object, the composition map $C_{Z}[-1] \rightarrow \mu \circ \pi(A)[-1] \rightarrow$ $N_{A}[-1]$ is the zero morphism (use that the morphism in (3.2) is trivial). Hence there is a non-trivial map $\phi_{1}: A \rightarrow N_{A}[-1]$ such that $\phi_{1} \circ l_{A} \circ \phi_{0}=\phi_{0}$. Now consider $A$ and $\phi_{1}$ instead of the pair $Z$ and $\phi_{0}$. Repeating the same argument as above we obtain another map $\phi_{2}: A \rightarrow N_{A}[-1]$ such that $\phi_{2} \circ l_{A} \circ \phi_{1}=\phi_{1}$. In conclusion, this procedure yields a sequence of morphisms $\phi_{i}: A \rightarrow N_{A}[-1]$ such that $\phi_{i+1} \circ l_{A} \circ \phi_{i}=\phi_{i}$, for $i>0$.

Set $a_{i}:=l_{A} \circ \phi_{i}$, for any $i>0$. This defines a sequence satisfying (3.1) in $\operatorname{End}(A)$. But by assumption this ring is idempotent noetherian. Hence there exists $n \in \mathbb{N}$ such that $a_{i} \circ \operatorname{End}(A)=a_{n} \circ \operatorname{End}(A)$, for all $i \geq n$. Given $N>n$, by Remark $3.2 a_{N}$ is idempotent. Since $a_{N}=l_{A} \circ \phi_{N}$ is not zero and $A$ is indecomposable, $a_{N}$ must be the identity and so $A$ is a direct summand of $N_{A}[-1]$. This implies $m_{A}=0$. Since $m_{A}$ corresponds to $\operatorname{id}_{\pi(A)}$ by adjunction, this means $\pi(A) \cong 0$ and so $\mu \circ \pi(A) \cong 0$ as well. This concludes the proof of Theorem 1.1.

Remark 3.4. It is important to note that the theorem above can be applied to a large class of triangulated categories. Assume that our triangulated category $\mathbf{S}$ is algebraic, i.e., it can be realized as a homotopy category of some differential graded category. If $\mathbf{S}$ is idempotent complete and equals to the closure of a set of objects $S$ under shifts, extensions and passage to direct factors (i.e., classically generated by this set), then by part b) of [6], Thm. 3.8, the category $\mathbf{S}$ is equivalent to a category of compact objects in the derived category of a dg-category, which is compactly generated and admits arbitrary direct sums. Thus it follows that if the rings of endomorphisms of all objects from $S$ are idempotent noetherian, then the statement of Theorem 1.1 holds for such $\mathbf{S}$.

3.2. Derived categories of abelian categories. Recall that an object $E$ in an abelian category is called noetherian if any ascending chain $G_{1} \subseteq G_{2} \subseteq \cdots \subseteq G_{n} \subseteq \cdots \subseteq E$ of subobjects of $E$ stabilizes, i.e., there is $n \in \mathbb{N}$ such that $G_{n}=G_{i}$ for all $i \geq n$. An 
abelian category is called noetherian if it is equivalent to a small category and every object is noetherian. An abelian category is called locally noetherian if it satisfies axiom (AB5) and has a set of noetherian generators (see, for example, [11]).

Remark 3.5. It can be proved that the full subcategory of noetherian objects in any locally noetherian abelian category is itself a noetherian abelian category.

The following statement says that the endomorphism algebra of a 'noetherian' object is idempotent noetherian.

Proposition 3.6. Let $\mathbf{A}$ be an abelian category with countable direct sums. Let $C \in \mathbf{D}^{\mathrm{b}}(\mathbf{A})$ be an object such that the cohomolgy $H^{k}(C) \in \mathbf{A}$ is noetherian for every $k \in \mathbb{Z}$. Then the algebra $\operatorname{End}_{\mathbf{D}^{\mathrm{b}}(\mathbf{A})}(C)$ is idempotent noetherian.

Proof. Let $\left\{a_{i}\right\}_{i \in \mathbb{N}}$ be a sequence in $\operatorname{End}(C)$ satisfying (3.1). We set $M:=\oplus_{i \in \mathbb{N}} C$ and $N:=\underset{\longrightarrow}{\longrightarrow}\left\{a_{i}\right\}$, so that there is a distinguished triangle in $\mathbf{D}^{\mathrm{b}}(\mathbf{A})$

$$
M \stackrel{f}{\longrightarrow} M \stackrel{a^{\prime}}{\longrightarrow} N
$$

where, denoting by $\iota_{i}: C \rightarrow M$ (for $i \in \mathbb{N}$ ) the inclusion of the $i$-th component, the morphism $f$ is defined by $f \circ \iota_{i}:=\iota_{i}-\iota_{i+1} \circ a_{i}$. By (3.1) the morphism $a: M \rightarrow C$ defined by $a \circ \iota_{i}:=a_{i}$ clearly satisfies $a \circ f=0$, hence there exists a morphism $b: N \rightarrow C$ such that $b \circ a^{\prime}=a$. Then, setting also $a_{i}^{\prime}:=a^{\prime} \circ \iota_{i}: C \rightarrow N$, we have

$$
b \circ a_{i}^{\prime}=a_{i} \quad \text { for all } i \in \mathbb{N} .
$$

Observe that if $i \in \mathbb{N}$ is such that $a_{i}^{\prime} \circ b: N \rightarrow N$ is an isomorphism, then $a_{i} \circ$ $\operatorname{End}(C)=b \circ \operatorname{Hom}(C, N)$. Indeed, by (3.4) we have $a_{i} \circ c=b \circ a_{i}^{\prime} \circ c$ for every $c \in \operatorname{End}(C)$. Conversely, again (3.4) implies that

$$
b \circ d=b \circ\left(a_{i}^{\prime} \circ b\right) \circ\left(a_{i}^{\prime} \circ b\right)^{-1} \circ d=a_{i} \circ b \circ\left(a_{i}^{\prime} \circ b\right)^{-1} \circ d
$$

for every $d \in \operatorname{Hom}(C, N)$.

Thus, in order to conclude that $\operatorname{End}(C)$ is idempotent noetherian, it is enough to prove that for $i \gg 0$ the morphism $a_{i}^{\prime} \circ b$ is an isomorphism in $\mathbf{D}^{\mathrm{b}}(\mathbf{A})$, which is the case if and only if $H^{k}\left(a_{i}^{\prime} \circ b\right)$ is an isomorphism in $\mathbf{A}$ for every $k \in \mathbb{Z}$. Since $C$ has only a finite number of non-zero cohomologies, we can fix $k$, and for simplicity of notation we will denote with an overline the functor $H^{k}$. Now, it is easy to see that the sequence

$$
0 \rightarrow \bar{M} \stackrel{\bar{f}}{\longrightarrow} \bar{M} \stackrel{\bar{a}}{\longrightarrow} \bar{C}
$$

is exact in A. On the other hand, the distinguished triangle (3.3) also yields an exact sequence

$$
0 \rightarrow \bar{M} \stackrel{\bar{f}}{\longrightarrow} \bar{M} \stackrel{\overline{a^{\prime}}}{\longrightarrow} \bar{N} \rightarrow 0 .
$$


Since $\bar{b} \circ \bar{a}^{\prime}=\bar{a}$, this implies that $\bar{b}: \bar{N} \rightarrow I:=\operatorname{im} \bar{a} \subseteq \bar{C}$ is an isomorphism. Denoting moreover im $\overline{a_{i}} \subseteq \bar{C}$ by $I_{i}$, (3.1) clearly implies that $I_{i} \subseteq I_{j}$ for $i<j$. As $\bar{C}$ is noetherian, there exists $n \in \mathbb{N}$ such that $I_{i}=I_{n}$ for $i \geq n$, and obviously $I_{n}=I$. Then we claim that $\overline{a_{i}^{\prime} \circ b}$ is an isomorphism for $i>n$. Indeed, this is equivalent to saying that $\overline{b \circ a_{i}^{\prime} \circ b}: \bar{N} \rightarrow I$ is an isomorphism. Since $\overline{b \circ a_{i}^{\prime}}=\overline{a_{i}}$ by (3.4), this is true if and only if $\left.\bar{a}_{i}\right|_{I}: I \rightarrow I$ is an isomorphism, which follows easily from the fact that $\overline{a_{i}} \circ \overline{a_{i-1}}=\overline{a_{i-1}}$ and $I_{i}=I_{i-1}=I$.

As a consequence we get the following.

Corollary 3.7. Let $\mathbf{A}$ be an abelian category with arbitrary direct sums and let $\mathbf{S} \subseteq \mathbf{D}^{\mathrm{b}}(\mathbf{A})$ be a thick full triangulated subcategory whose objects have noetherian cohomology. Let $\mathrm{F}: \mathbf{S} \rightarrow \mathbf{T}$ be a full exact functor to a triangulated category $\mathbf{T}$. Then there is an orthogonal decomposition

$$
\mathbf{S}=(\operatorname{ker} F)^{\perp} \oplus \operatorname{ker} F
$$

and $\left.\mathrm{F}\right|_{(\mathrm{ker} F) \perp}$ is faithful.

Proof. As in Remark 3.4, by part b) of [6], Thm. 3.8, the category $\mathbf{S}$ (which is idempotent complete being a thick subcategory of an idempotent complete category) is equivalent to a category of compact objects in the derived category of a dg-category. Thus Theorem 1.1 and Proposition 3.6 give the desired conclusion.

Remark 3.8. If $\mathbf{A}$ is a locally noetherian abelian category and $\mathbf{S}$ is the full subcategory of $\mathrm{D}^{\mathrm{b}}(\mathbf{A})$ consisting of all objects with noetherian cohomology, then, in view of Remark $3.5, \mathbf{S}$ is automatically a thick triangulated subcategory and Corollary 3.7 applies.

\section{The geometric case}

Let $X$ be a noetherian scheme. We denote by $\mathbf{D}(X)$ the full subcategory of the derived category of sheaves of $\mathcal{O}_{X}$-modules consisting of (unbounded) complexes with quasi-coherent cohomology. Let $\mathrm{D}^{\mathrm{b}}(X)$ be the full subcategory of $\mathbf{D}(X)$ consisting of bounded complexes with coherent cohomology. Being $X$ noetherian, $\mathrm{D}^{\mathrm{b}}(X)$ is equivalent to $\mathbf{D}^{\mathrm{b}}(\boldsymbol{c o h}(X))$, where $\operatorname{coh}(X)$ is the abelian category of coherent sheaves on $X$ (see [1], Cor. 2.2.2.2). Moreover, $\operatorname{Perf}(X)$ will be the full subcategory of $\mathbf{D}(X)$ consisting of perfect complexes. Notice that $\operatorname{Perf}(X) \subseteq \mathrm{D}^{\mathrm{b}}(X)$.

Now assume that $Z$ is a closed subscheme of $X$. We denote by $\mathbf{D}_{Z}(X)$ the full subcategory of $\mathbf{D}(X)$ consisting of complexes with cohomology supported on $Z$. We will also need the following full subcategories of $\mathbf{D}_{Z}(X)$ :

$$
\mathrm{D}_{Z}^{\mathrm{b}}(X):=\mathbf{D}_{Z}(X) \cap \mathrm{D}^{\mathrm{b}}(X), \quad \operatorname{Perf}_{Z}(X):=\mathbf{D}_{Z}(X) \cap \operatorname{Perf}(X) .
$$


Proposition 4.1 ([12], Theorem 6.8). The category $\mathbf{D}_{Z}(X)$ is compactly generated, and the category of compact objects $\mathbf{D}_{Z}(X)^{\mathrm{c}}$ coincides with $\operatorname{Perf}_{Z}(X)$.

Remark 4.2. The category $\mathbf{Q} \operatorname{coh}(X)$ of quasi-coherent sheaves of $\mathcal{O}_{X}$-modules over a noetherian scheme $X$ is a locally noetherian abelian category and the full subcategory of noetherian objects in $\mathbf{Q} \operatorname{coh}(X)$ is precisely $\operatorname{coh}(X)$. The same is true in the supported case as well.

The following result is then a straightforward consequence of Proposition 3.6.

Proposition 4.3. If $X$ is a noetherian scheme containing a closed subscheme $Z$ and $\mathscr{E} \in \mathrm{D}_{Z}^{\mathrm{b}}(X)$, then the endomorphism ring $\operatorname{End}_{\mathrm{D}_{Z}^{\mathrm{b}}(X)}(\mathcal{E})$ is idempotent noetherian.

Corollary 3.7 (applied to the case $\mathbf{A}=\mathbf{Q c o h}_{Z}(X)$ ) and Remark 4.2 immediately give the following.

Corollary 4.4. Let $X$ be a noetherian scheme containing a closed subscheme $Z$. If $\mathbf{S}$ is either $\operatorname{Perf}_{Z}(X)$ or $\mathrm{D}_{Z}^{\mathrm{b}}(X)$ and $\mathrm{F}: \mathbf{S} \rightarrow \mathbf{T}$ is a full exact functor to a triangulated category $\mathbf{T}$, then there is an orthogonal decomposition $\mathbf{S}=(\operatorname{ker} \mathrm{F})^{\perp} \oplus \operatorname{ker} \mathrm{F}$ and $\left.\mathrm{F}\right|_{(\mathrm{ker} F)^{\perp}}$ is faithful.

Consider now the following rather general result.

Lemma 4.5. Let $\mathbf{T}$ be a compactly generated triangulated category with arbitrary direct sums such that $\mathbf{T}^{\mathrm{c}}$ has an orthogonal decomposition $\mathbf{T}^{\mathrm{c}}=\mathbf{S}_{1} \oplus \mathbf{S}_{2}$. Then $\mathbf{T}$ has an orthogonal decomposition $\mathbf{T}=\widetilde{\mathbf{S}}_{1} \oplus \widetilde{\mathbf{S}}_{2}$, where $\widetilde{\mathbf{S}}_{i}$, for $i=1,2$, is the localizing subcategory generated by $\mathbf{S}_{i}$.

Proof. We first show that $\widetilde{\mathbf{S}}_{1}$ and $\widetilde{\mathbf{S}}_{2}$ are orthogonal. Indeed, if $A \in \mathbf{S}_{1}$, then $A^{\perp}:=$ $\{B \in \mathbf{T} \mid \operatorname{Hom}(A, B)=0\} \supseteq \widetilde{\mathbf{S}}_{2}$ because $A^{\perp}$ is localizing, being $A$ compact. On the other hand, if $B \in \widetilde{\mathbf{S}}_{2}$, then ${ }^{\perp} B:=\{A \in \mathbf{T} \mid \operatorname{Hom}(A, B)=0\} \supseteq \mathbf{S}_{1}$ by what we have just proved. Since ${ }^{\perp} B$ is a localizing subcategory of $\mathbf{T}$, this implies that ${ }^{\perp} B \supseteq$ $\widetilde{\mathbf{S}}_{1}$. Hence $\operatorname{Hom}\left(\widetilde{\mathbf{S}}_{1}, \widetilde{\mathbf{S}}_{2}\right)=0$ and a similar argument yields $\operatorname{Hom}\left(\widetilde{\mathbf{S}}_{2}, \widetilde{\mathbf{S}}_{1}\right)=0$.

For $i=1,2$, the canonical full embedding $j_{i}: \widetilde{\mathbf{S}}_{i} \rightarrow \mathbf{T}$ has a right adjoint $s_{i}: \mathbf{T} \rightarrow \widetilde{\mathbf{S}}_{i}$ (see, for example, Theorem 8.3.3 and Proposition 8.4.2 in [8]). This provides a canonical map $j_{1} \circ s_{1}(X) \oplus j_{2} \circ s_{2}(X) \rightarrow X$, for any $X \in \mathbf{T}$, which sits in a distinguished triangle

$$
C_{X}[-1] \rightarrow j_{1} \circ s_{1}(X) \oplus j_{2} \circ s_{2}(X) \rightarrow X \rightarrow C_{X} .
$$

For any compact object $S \in \mathbf{S}_{1}$, applying the functor $\operatorname{Hom}\left(j_{1}(S),-\right)$ to this triangle, we obtain isomorphisms

$\operatorname{Hom}\left(j_{1}(S), j_{1} \circ s_{1}(X) \oplus j_{2} \circ s_{2}(X)\right) \stackrel{\sim}{\longrightarrow} \operatorname{Hom}\left(S, s_{1}(X)\right) \stackrel{\sim}{\longrightarrow} \operatorname{Hom}\left(j_{1}(S), X\right)$. 
This implies that $\operatorname{Hom}\left(j_{i}(S), C_{X}\right)=0$ for any compact object $S \in \mathbf{S}_{i}$ and $i=1,2$. Since $\mathbf{T}$ is compactly generated and, by assumption, any compact object of $\mathbf{T}$ is a direct sum of objects from $\mathbf{S}_{1}$ and $\mathbf{S}_{2}$, we deduce that $C_{X}=0$. Hence the map $j_{1} \circ s_{1}(X) \oplus j_{2} \circ s_{2}(X) \rightarrow X$ is an isomorphism.

We can now apply the previous result to a concrete geometric question.

Corollary 4.6. Let $Z$ be a connected closed subscheme of a quasi-compact quasiseparated scheme $X$. Then the triangulated categories $\operatorname{Perf}_{Z}(X), \mathrm{D}_{Z}^{\mathrm{b}}(X)$, and $\mathbf{D}_{Z}(X)$ are indecomposable.

Proof. By Lemma 4.5 and Proposition 4.1, a non-trivial orthogonal decomposition of $\operatorname{Perf}_{Z}(X)$ induces a non-trivial orthogonal decomposition of $\mathbf{D}_{Z}(X)$. So it is enough to show that the latter category and $\mathrm{D}_{Z}^{\mathrm{b}}(X)$ are indecomposable. As the proof for these two categories is the same, we will deal only with $\mathbf{D}_{Z}(X)$.

Hence assume that there exists an orthogonal decomposition $\mathbf{D}_{Z}(X)=\mathbf{S}_{1} \oplus \mathbf{S}_{2}$. Following the strategy in [4], Example 3.2, consider the structure sheaf $\mathcal{O}_{Z}$ of the subscheme $Z \subseteq X$. Since $Z$ is connected, the object $\mathcal{O}_{Z}$ is indecomposable in $\mathbf{D}_{Z}(X)$ and thus it belongs to one of the categories $\mathbf{S}_{i}$, for $i=1,2$. Without loss of generality, let it belong to $\mathbf{S}_{1}$.

For any closed point $z \in Z$, there is a non-trivial morphism $\mathcal{O}_{Z} \rightarrow \mathcal{O}_{z}$. Thus $\mathcal{O}_{z} \in \mathbf{S}_{1}$, for all closed point $z \in Z$. Finally, consider a perfect complex $\mathcal{A} \in$ $\operatorname{Perf}_{Z}(X)$. Take an affine open subset $U \cong \operatorname{Spec}(A) \subseteq X$ such that the restriction of $\mathcal{A}$ to $U$ is a non-trivial object. By definition, $\left.\mathcal{A}\right|_{U}$ is isomorphic in $\mathbf{D}(U)$ to an object $\mathcal{P}$ corresponding to a bounded complex of finitely generated projective $A$-modules $P$. Set $i$ such that $H^{i}(P)$ is the greatest non-trivial cohomology of $P$. Then $H^{i}(P)$ is a finitely generated $A$-module and, by Nakayama's lemma, there is a non-trivial map $H^{i}(\mathcal{P}) \rightarrow \mathcal{O}_{z}$, for a closed point $z \in Z$. This induces a non-trivial map $\mathcal{P} \rightarrow \mathcal{O}_{z}$ and therefore all perfect complexes belong to $\mathbf{S}_{1}$. This implies that $\mathbf{S}_{1}$ coincides with $\mathbf{D}_{Z}(X)$.

This result, combined with Corollary 4.4, gives Theorem 1.2.

\section{A counterexample}

In this section we provide an example of a full exact and non-trivial functor $\mathrm{F}: \mathbf{T}_{1} \rightarrow$ $\mathbf{T}_{2}$ between triangulated categories such that $\mathbf{T}_{1}$ is indecomposable and $\mathrm{F}$ is not faithful.

To this end, let $A$ be a commutative algebra over a field $\mathbb{k}$ with generators $x_{1}, x_{2}, \ldots$ and with relations $x_{j} x_{i}=x_{i}$ for $i<j$. Let Mod- $A$ be the category of right $A$-modules and set $\mathbf{D}(A):=\mathbf{D}(\operatorname{Mod}-A)$. Denote by $\operatorname{Perf}(A)$ the full subcategory of $\mathbf{D}(A)$ of perfect complexes, i.e., the smallest thick subcategory of $\mathbf{D}(A)$ containing $A$. 
Lemma 5.1. The triangulated category $\operatorname{Perf}(A)$ is indecomposable.

Proof. Obviously $\operatorname{Perf}(A) \cong \operatorname{Perf}(\operatorname{Spec}(A))$. By Corollary 4.6, the result follows once we know that $\operatorname{Spec}(A)$ is connected. This, in turn, is equivalent to showing that $A$ does not contain non-trivial idempotents. But this is an easy exercise using the definition of the algebra $A$.

Denote by $I$ the ideal generated by all $x_{i}$ so that $A / I \cong \mathbb{k}$. Consider the functor

$$
\mathrm{G}: \mathbf{D}(A) \rightarrow \mathbf{D}(\mathbb{k}), \quad X \mapsto X \otimes_{A}^{\mathbf{L}} \mathbb{k},
$$

and set $\mathrm{F}:=\left.\mathrm{G}\right|_{\operatorname{Perf}(A)}: \operatorname{Perf}(A) \rightarrow \mathbf{D}(\mathbb{k})$.

Lemma 5.2. The functor $\mathrm{F}$ is full.

Proof. It is easy to see that the result follows if we prove that the morphisms

$$
\begin{aligned}
& \operatorname{Hom}_{A}(A, P) \rightarrow \operatorname{Hom}_{\mathbb{k}}(\mathrm{F}(A), \mathrm{F}(P))=\operatorname{Hom}_{\mathbb{k}}\left(\mathbb{k}, P \otimes_{A}^{\mathbf{L}} \mathbb{k}\right), \\
& \operatorname{Hom}_{A}(P, A) \rightarrow \operatorname{Hom}_{\mathbb{k}}(\mathrm{F}(P), \mathrm{F}(A))=\operatorname{Hom}_{\mathbb{k}}\left(P \otimes_{A}^{\mathbf{L}} \mathbb{k}, \mathbb{k}\right)
\end{aligned}
$$

are surjective for any $P \in \operatorname{Perf}(A)$. Any perfect complex $P$ is a direct summand in $\operatorname{Perf}(A)$ of a bounded complex of finitely generated free $A$-modules. Hence, it is sufficient to consider the case when $P$ itself is quasi-isomorphic to a bounded complex

$$
Q^{\bullet}=\left\{Q^{t} \stackrel{d^{t}}{\longrightarrow} \cdots \rightarrow Q^{-1} \stackrel{d^{-1}}{\longrightarrow} Q^{0} \stackrel{d^{0}}{\longrightarrow} Q^{1} \stackrel{d^{1}}{\longrightarrow} \cdots \stackrel{d^{s-1}}{\longrightarrow} Q^{s}\right\}
$$

of finitely generated free $A$-modules.

Take a morphism $f_{1}: \mathbb{k} \rightarrow Q^{0} \otimes_{A} \mathbb{k}$ such that the composition $\left(d^{0} \otimes \mathbb{k}\right) \circ f_{1}$ is trivial. Composing with $A \rightarrow \mathbb{k}$, the morphism $f_{1}$ induces a map $g_{1}: A \rightarrow Q^{0} \otimes_{A} \mathbb{k}$ which, in turn, lifts to $h_{1}: A \rightarrow Q^{0}$. Now the element $\left(d^{0} \circ h_{1}\right)(1) \in Q^{1} \cong A^{m}$ is in $I^{m}$ and $x_{n}\left(d^{0} \circ h_{1}\right)(1)=\left(d^{0} \circ h_{1}\right)(1)$ for a sufficiently large $n$. So setting $h_{1}^{\prime}:=\left(1-x_{n}\right) \circ h_{1}$, we get $d^{0} \circ h_{1}^{\prime}(1)=0$ and $\mathrm{F}\left(h_{1}^{\prime}\right)=f_{1}$. In particular, the first morphism in (5.1) is surjective.

Similarly, to deal with the second morphism in (5.1), let $f_{2}: Q^{0} \otimes_{A} \mathbb{k} \rightarrow \mathbb{k}$ be a morphism such that the composition $f_{2} \circ\left(d^{-1} \otimes \mathbb{k}\right)$ is trivial. Again, composing with the natural morphism $Q^{0}=Q^{0} \otimes_{A} A \rightarrow Q^{0} \otimes_{A} \mathbb{k}$, we get a morphism $g_{2}: Q^{0} \rightarrow \mathbb{k}$ which lifts to a morphism $h_{2}: Q^{0} \rightarrow A$. For very large $n$, define $h_{2}^{\prime}:=\left(1-x_{n}\right) \circ h_{2}$ so that, again, $h_{2}^{\prime} \circ d^{-1}\left(a_{j}\right)=0$, for all $a_{j}$ in the set of generators $a_{1}, \ldots a_{r}$ of $Q^{-1}$. Then $\mathrm{F}\left(h_{2}^{\prime}\right)=f_{2}$, and this concludes the proof.

To prove that $\mathrm{F}$ is not faithful, consider the non-trivial morphism $x_{i}: A \rightarrow A$ for $i$ any positive integer. On the other hand, the morphism $\mathrm{F}\left(x_{i}\right): \mathbb{k} \rightarrow \mathbb{k}$ is the trivial morphism. 
Acknowledgements. D. O. thanks Simons Center for Geometry and Physics for hospitality and stimulating atmosphere. Part of this article was written while P. S. was visiting the Institut Henri Poincaré in Paris whose warm hospitality is gratefully acknowledged. P. S. would like to dedicate the paper to his wife Anna and to his daughter Giulia who came to light during the final write-up of the article.

\section{References}

[1] P. Berthelot, A. Grothendieck, L. Illusie (eds.), Théorie des intersections et théorème de Riemann-Roch. Séminaire de géométrie algébrique du Bois Marie 1966-67 (SGA 6), Lecture Notes in Math. 225, Springer-Verlag, Berlin 1971. Zbl 0218.14001 MR 0354655

[2] A. Bondal and D. Orlov, Semiorthogonal decomposition for algebraic varieties. Preprint 1995. arXiv:alg-geom/9506012

[3] A. Bondal and M. van den Bergh, Generators and representability of functors in commutative and noncommutative geometry. Moscow Math. J. 3 (2003), no. 1, 1-36. Zbl 1135.18302 MR 1996800

[4] T. Bridgeland, Equivalences of triangulated categories and Fourier-Mukai transforms. Bull. London Math. Soc. 31 (1999), 25-34. Zbl 0937.18012 MR 1651025

[5] A. Canonaco and P. Stellari, Twisted Fourier-Mukai functors. Adv. Math. 212 (2007), 484-503. Zbl 1116.14009 MR 2329310

[6] B. Keller, On differential graded categories. In Proc. Internat. Congr. Mathematicians, vol. II, European Math. Soc. Publ. House, Zürich 2006, 151-190. Zbl 1140.18008 MR 2275593

[7] A. Neeman, The connection between the $K$-theory localization theorem of Thomason, Trobaugh and Yao and the smashing subcategories of Bousfield and Ravenel. Ann. Sci. École Norm. Sup. (4) 25 (1992), 547-566. Zbl 0868.19001 MR 1191736

[8] A. Neeman, Triangulated categories. Ann. of Math. Stud. 148, Princeton University Press, Princeton, NJ, 2001. Zbl 0974.18008 MR 1812507

[9] D. O. Orlov, Equivalences of derived categories and K3 surfaces. J. Math. Sci. 84 (1997), 1361-1381. Zbl 0938.14019 MR 1465519

[10] D. O. Orlov, Triangulated categories of singularities and D-branes in Landau-Ginzburg models. Tr. Mat. Inst. Steklova 246 (2004), 240-262; English transl. Proc. Steklov Inst. Math. 246 (2004), 227-248. Zbl 1101.81093 MR 2101296

[11] N. Popescu, Abelian categories with applications to rings and modules. London Math.1 Soc. Monogr 3, Academic Press, London 1973. Zbl 0271.18006 MR 0340375

[12] R. Rouquier, Dimensions of triangulated categories. J. K-Theory 1 (2008), 193-256. Zbl 1165.18008 MR 2434186

Received March 10, 2011 
A. Canonaco, Dipartimento di Matematica "F. Casorati”, Università degli Studi di Pavia, Via Ferrata 1, 27100 Pavia, Italy

E-mail: alberto.canonaco@unipv.it

D. Orlov, Algebraic Geometry Section, Steklov Mathematical Institute RAS, 8 Gubkin Str., Moscow 119991, Russia

E-mail: orlov@mi.ras.ru

P. Stellari, Dipartimento di Matematica "F. Enriques", Università degli Studi di Milano, Via Cesare Saldini 50, 20133 Milano, Italy

E-mail: paolo.stellari@unimi.it 